\title{
Travelling Wave Solutions for Nonlinear Schrödinger Equation with a Higher-Order Dispersive Term
}

\author{
Rui Cao ${ }^{1,2}$ \\ ${ }^{1}$ Department of Mathematics, Heze University, Heze 274000, China \\ ${ }^{2}$ College of Mathematics and Software Science, Sichuan Normal University, Chengdu 610066, China
}

Correspondence should be addressed to Rui Cao; ruicao999@126.com

Received 12 July 2013; Accepted 10 September 2013

Academic Editor: Yong Hong Wu

Copyright (C) 2013 Rui Cao. This is an open access article distributed under the Creative Commons Attribution License, which permits unrestricted use, distribution, and reproduction in any medium, provided the original work is properly cited.

\begin{abstract}
A nonlinear Schrödinger equation with a higher-order dispersive term describing the propagation of ultrashort femtosecond pulses in optical fibres is considered and is transformed into a second-order nonlinear ordinary differential equation. We investigate the exact travelling wave solutions of the nonlinear Schrödinger equation using three methods, namely, the auxiliary equation method, the first integral method, and the direct integral method. As a result, Jacobi elliptic function solution, hyperbolic function solution, trigonometric function solution, and rational solution with parameters are obtained successfully. When the parameters are taken as special values, the two known solitary wave solution and periodic wave solution are derived from the solutions obtained. The aim of the paper is to compare the efficiency of the three methods.
\end{abstract}

\section{Introduction}

We are concerned with the following nonlinear Schrödinger equation with a higher-order dispersive term [1]:

$$
\begin{aligned}
i \psi_{x}+ & \alpha_{1} \psi_{t t}+\alpha_{2} \psi|\psi|^{2}-i \alpha_{3} \psi_{t t t} \\
& -i \alpha_{4}\left(\psi|\psi|^{2}\right)_{t}-i \alpha_{5}\left(|\psi|^{2}\right)_{t} \psi=0,
\end{aligned}
$$

where $\psi=\psi(x, t)$ is slowly varying envelop of the electric field, the subscripts $x$ and $t$ are the spatial and temporal partial derivative in retard time coordinates, and $\alpha_{1}$, $\alpha_{2}, \alpha_{3}, \alpha_{4}$, and $\alpha_{5}$ are real parameters related to the group velocity, self-phase modulation, third-order dispersion, selfsteepening, and self-frequency shift arising from stimulated Raman scattering, respectively. Equation (1) can be applied to describe the propagation of ultrashort femtosecond pulses in optical fibres.

The nonlinear Schrödinger (NLS) equations occur in many branches of physics such as nonlinear optics, quantum mechanics, condensed matter physics, and plasma physics $[2,3]$. There have been various Schrödinger-type equations in the study of nonlinear phenomena. All these phenomena can be better understood with the help of exact analytical solutions. Recently, a considerable amount of research work has been devoted to searching for exact solution of NLS equation with a variety of nonlinearities. For example, $\mathrm{Li}$ and Wang [4] applied the $\left(G^{\prime} / G\right)$-expansion method to study the higher-order nonlinear Schrödinger equation (1) and obtained travelling wave solutions under constraint condition. Ma and Lee [5] proposed the transformed rational function method for solving the $(3+1)$-dimensional JimboMiwa equation and obtained exact solutions. Wu and Dai [6] investigated quintic nonlinear derivative Schrödinger equation and obtained soliton solutions including chirped bright, dark, and kink soliton solutions with the aid of a special auxiliary equation. Ma [7] introduced a class of bilinear differential operators which describe generalized bilinear differential equations and discussed their links with the Bell polynomial. More details are presented in $[8,9]$.

Exact solutions (especially travelling wave solution) of nonlinear evolution equation (NLEE) play an important role in the study of nonlinear physical phenomenon $[2,10]$. During the past decades, various analytical and numerical methods for constructing the travelling wave solutions to NLEE have been proposed, such as Hirota's bilinear method $[11,12]$, the Bäcklund transformation method [13], the sinecosine method [14], the tanh-function method $[15,16]$, the 
Exp-function method $[17,18]$, the Jacobi elliptic expansion method [19], the first integral method [20], and the Riccati equation method $[21,22]$. The reader is referred to $[1,23,24]$ and the references therein.

In this paper, our main purpose is to investigate the travelling wave solutions for nonlinear Schrödinger equation with a higher-order dispersive term (1). The rest of the paper is organized as follows. In Section 2, we describe these methods for solving nonlinear evolution equations including the auxiliary equation method, the first integral method, and the direct integral method. In Section 3, we apply these methods to (1) and obtain enveloped travelling wave solutions. Finally, the concluding remarks are presented in Section 4.

\section{Description of Methods}

2.1. The Auxiliary Equation Method. For a given nonlinear partial differential equation with a physical field $u(x, t)$ in two independent variables $x, t$,

$$
H\left(u, u_{t}, u_{x}, u_{t t}, u_{t x}, u_{x x}, \ldots\right)=0 .
$$

Step 1. Making use of the travelling wave transformation,

$$
u(x, t)=u(\xi), \quad \xi=k(x-v t) .
$$

Then, (2) is reduced to the following ordinary differential equation (ODE):

$$
Q\left(u,-k v u^{\prime}, k u^{\prime}, k^{2} v^{2} u^{\prime \prime},-k^{2} v u^{\prime \prime}, k^{2} u^{\prime \prime}, \ldots\right)=0 .
$$

Step 2. We introduce a new ansatz:

$$
\begin{aligned}
u(\xi)=a_{0}+\sum_{i=1}^{N}[ & a_{i} \phi^{i}(\xi)+b_{i} \phi^{i-1}(\xi)\left(R+\phi^{2}(\xi)\right)^{1 / 2} \\
& \left.+c_{i} \phi^{-i}(\xi)\right],
\end{aligned}
$$

where $N$ is a positive integer and $a_{0}, a_{i}, b_{i}$, and $c_{i}$ are constants, while the new variable $\phi=\phi(\xi)$ satisfies the Riccati equation [21]:

$$
\phi^{\prime}=\frac{d \phi}{d \xi}=R+\phi^{2}(\xi) .
$$

Step 3. We define the degree of $u(\xi)$ as $D[u(\xi)]=N$. The degree of $u^{(p)}(\xi)$ can be calculated by

$$
\begin{gathered}
D\left[u^{(p)}\right]=N+p, \\
D\left[u^{p}\left(u^{(q)}\right)^{s}\right]=N p+(N+q) s .
\end{gathered}
$$

Step 4. Substituting (5) along with (6) into (4) and setting all coefficients of $\phi^{j}(\xi){\sqrt{R+\phi^{2}(\xi)}}^{m}(j=0,1, \ldots, m=0,1)$ to zero, we get a system of nonlinear algebraic equations.

Step 5. Solving the overdetermined system in Step 4 by the symbolic computation system Mathematica, we obtain travelling wave solutions of (1).
Remark 1. One of the main steps in the method is to determine the value of $N$ in (5). The value of $N$ can be determined by considering the homogeneous balance between the highest-order derivative and nonlinear term appearing in (4). If $N$ is not a positive integer, then we first make the transformation $u=\psi^{N}$.

2.2. The First Integral Method. The nonlinear partial differential equation in two independent variables $x, t$

$$
F\left(u, u_{t}, u_{x}, u_{t t}, u_{t x}, u_{x x}, \ldots\right)=0
$$

can be transformed into a nonlinear ODE

$$
G\left(u,-k v u^{\prime}, k u^{\prime}, k^{2} v^{2} u^{\prime \prime},-k^{2} v u^{\prime \prime}, k^{2} u^{\prime \prime}, \ldots\right)=0,
$$

under the wave transformation $u(x, t)=u(\xi)$, where the wave variable $\xi=k(x-v t)$.

Next, we introduce a new independent variable

$$
X(\xi)=u(\xi), \quad Y(\xi)=\frac{d X(\xi)}{d \xi}=\dot{X}(\xi),
$$

which leads (9) to a system of nonlinear ODEs

$$
\begin{gathered}
\dot{X}(\xi)=Y(\xi), \\
\dot{Y}(\xi)=P(X(\xi), Y(\xi)) .
\end{gathered}
$$

By the qualitative theory of ordinary differential equations, if we can find the integrals to (11), then the general solutions to (11) can be obtained directly. In order to obtain one first integral of (11), we will apply the Division Theorem.

\section{Application}

In this section, we will illustrate three methods mentioned above and obtain the exact travelling wave solutions of (1).

Assume that the solution of (1) can be written as

$$
\psi(x, t)=u(\xi) \exp [i(k x-\omega t)], \quad \xi=t-\lambda x+\xi_{0},
$$

where $k, \omega$, and $\lambda$ are constants to be determined. Substituting (12) into (1) and separating the real and imaginary parts, we obtain the ODEs for $u(\xi)$ :

$$
\alpha_{3} u^{\prime \prime \prime}+\left(3 \alpha_{4}+2 \alpha_{5}\right) u^{2} u^{\prime}-\left(3 \alpha_{3} \omega^{2}-\lambda-2 \alpha_{1} \omega\right) u^{\prime}=0,
$$

$$
\left(\alpha_{1}-3 \alpha_{3} \omega\right) u^{\prime \prime}+\left(\alpha_{2}-\alpha_{4} \omega\right) u^{3}+\left(\alpha_{3} \omega^{3}-\alpha_{1} \omega^{2}-k\right) u=0
$$

Integrating (13) once and setting the constant of integration to zero, we get

$$
\alpha_{3} u^{\prime \prime}-\left(3 \alpha_{3} \omega^{2}-\lambda-2 \alpha_{1} \omega\right) u+\frac{\left(3 \alpha_{4}+2 \alpha_{5}\right)}{3} u^{3}=0 .
$$

Under the conditions

$$
\begin{gathered}
\omega=\frac{\alpha_{1}\left(3 \alpha_{4}+2 \alpha_{5}\right)-3 \alpha_{2} \alpha_{3}}{6 \alpha_{3}\left(\alpha_{4}+\alpha_{5}\right)}, \\
k=8 \alpha_{1} \omega^{2}-8 \alpha_{3} \omega^{3}-\frac{2 \alpha_{1}^{2} \omega+\alpha_{1} \lambda}{\alpha_{3}}+3 \lambda \omega,
\end{gathered}
$$


(14) and (15) are transformed into the following equation:

$$
u^{\prime \prime}+A u+B u^{3}=0
$$

where $A$ and $B$ are defined by $A=\left(\lambda+2 \alpha_{1} \omega-3 \alpha_{3} \omega^{2}\right) / \alpha_{3}$, and $B=\left(3 \alpha_{4}+2 \alpha_{5}\right) / 3 \alpha_{3}$.

3.1. Using the Auxiliary Equation Method to Solve (1). Considering the homogeneous balance between $u^{\prime \prime}$ and $u^{3}$ in (17) yields $N=1$. So we assume that (1) has the following formal solutions:

$$
u(\xi)=a_{0}+a_{1} \phi(\xi)+b_{1} \sqrt{R+\phi^{2}(\xi)}+c_{1} \phi^{-1}(\xi)
$$

where $a_{0}, a_{1}, b_{1}$, and $c_{1}$ are constants to be determined.

Substituting (18) along with (6) into (17), collecting the coefficients of $\phi^{i}(\xi)\left(\sqrt{R+\phi^{2}(\xi)}\right)^{j}(i=-3,-2,-1,0,1,2,3$, $j=0,1)$, and setting each coefficient to zero, one obtains the system of algebraic equations. Then solving the obtained algebraic equations, we have a set of nontrivial solutions.

Case 1. One has

$$
\begin{aligned}
& a_{0}=0, \quad b_{1}=0, \quad c_{1}=0, \\
& a_{1}= \pm \sqrt{-\frac{2}{B}}, \quad 2 R+A=0 .
\end{aligned}
$$

Case 2. One has

$$
\begin{aligned}
& a_{0}=0, \quad c_{1}=0, \quad a_{1}= \pm \sqrt{-\frac{1}{2 B}}, \\
& b_{1}=\mp \sqrt{-\frac{1}{2 B}}, \quad R+2 A=0 .
\end{aligned}
$$

Case 3. One has

$$
\begin{aligned}
& a_{0}=0, \quad b_{1}=0, \quad a_{1}= \pm \sqrt{-\frac{2}{B}}, \\
& c_{1}= \pm \frac{A}{2} \sqrt{-\frac{1}{2 B}}, \quad A-4 R=0 .
\end{aligned}
$$

From the expression in (12), (19), (20), (21) and the solutions of (6), we derive the following travelling wave solutions of (1).

Family 1. If $R<0, A>0, \alpha_{3}\left(3 \alpha_{4}+2 \alpha_{5}\right)<0$, then

$$
\begin{aligned}
& \psi_{1,1}= \pm \sqrt{-\frac{A}{B}} \operatorname{coth}\left[\sqrt{\frac{A}{2}}\left(t-\lambda x+\xi_{0}\right)\right] \exp [i(k x-\omega t)] \\
& \psi_{1,2}= \pm \sqrt{-\frac{A}{B}} \tanh \left[\sqrt{\frac{A}{2}}\left(t-\lambda x+\xi_{0}\right)\right] \exp [i(k x-\omega t)]
\end{aligned}
$$

$$
\begin{aligned}
& \psi_{1,3}= \pm \sqrt{-\frac{A}{B}} {\left[\tanh \left[\sqrt{2 A}\left(t-\lambda x+\xi_{0}\right)\right]\right.} \\
&\left.+i \operatorname{sech}\left[\sqrt{2 A}\left(t-\lambda x+\xi_{0}\right)\right]\right] \\
& \times \exp [i(k x-\omega t)], \\
& \psi_{1,4}= \pm \sqrt{-\frac{A}{B}}\left[\operatorname{coth}\left[\sqrt{2 A}\left(t-\lambda x+\xi_{0}\right)\right]\right. \\
&\left.\quad+\operatorname{csch}\left[\sqrt{2 A}\left(t-\lambda x+\xi_{0}\right)\right]\right] \\
& \quad \times \exp [i(k x-\omega t)],
\end{aligned}
$$

where $\psi_{1,1}$ and $\psi_{1,2}$ are the known solitary wave solution found by Liu (formulae (30) and (31) in [23]).

Family 2. If $R>0, A>0, \alpha_{3}\left(3 \alpha_{4}+2 \alpha_{5}\right)<0$, then

$$
\begin{aligned}
& \psi_{1,5}= \pm \sqrt{-\frac{A}{2 B}}\left[\cot \left[\sqrt{\frac{A}{4}}\left(t-\lambda x+\xi_{0}\right)\right]\right. \\
& \left.+\tan \left[\sqrt{\frac{A}{4}}\left(t-\lambda x+\xi_{0}\right)\right]\right] \exp [i(k x-\omega t)] .
\end{aligned}
$$

Family 3. If $R>0, A<0, \alpha_{3}\left(3 \alpha_{4}+2 \alpha_{5}\right)<0$, then

$$
\begin{aligned}
& \psi_{1,6}= \pm \sqrt{\frac{A}{B}} \tan \left[\sqrt{-\frac{A}{2}}\left(t-\lambda x+\xi_{0}\right)\right] \exp [i(k x-\omega t)], \\
& \psi_{1,7}= \pm \sqrt{\frac{A}{B}} \cot \left[\sqrt{-\frac{A}{2}}\left(t-\lambda x+\xi_{0}\right)\right] \exp [i(k x-\omega t)] \\
& \psi_{1,8}= \pm \sqrt{\frac{A}{B}}\left[\tan \left[\sqrt{-2 A}\left(t-\lambda x+\xi_{0}\right)\right]\right. \\
& \left.-\sec \left[\sqrt{-2 A}\left(t-\lambda x+\xi_{0}\right)\right]\right] \\
& \times \exp [i(k x-\omega t)] \text {, } \\
& \psi_{1,9}= \pm \sqrt{\frac{A}{B}}\left[\cot \left[\sqrt{-2 A}\left(t-\lambda x+\xi_{0}\right)\right]\right. \\
& \left.+\csc \left[\sqrt{-2 A}\left(t-\lambda x+\xi_{0}\right)\right]\right] \\
& \times \exp [i(k x-\omega t)] \text {, }
\end{aligned}
$$

where $\psi_{1,6}$ and $\psi_{1,7}$ are the known periodic wave solution obtained in [23] (formulae (34) and (35) in [23]).

Family 4. If $R<0, A<0, \alpha_{3}\left(3 \alpha_{4}+2 \alpha_{5}\right)<0$, then

$$
\begin{aligned}
& \psi_{1,10} \\
& = \pm \sqrt{\frac{A}{2 B}}\left[\tanh \left[\sqrt{-\frac{A}{4}}\left(t-\lambda x+\xi_{0}\right)\right]\right. \\
& \left.-\operatorname{coth}\left[\sqrt{-\frac{A}{4}}\left(t-\lambda x+\xi_{0}\right)\right]\right] \exp [i(k x-\omega t)] .
\end{aligned}
$$


Family 5. If $R=0, \alpha_{3}\left(3 \alpha_{4}+2 \alpha_{5}\right)<0$, then we obtain the rational solution of (1):

$$
\begin{aligned}
\psi_{1,11}= & \pm \sqrt{-\frac{6 \alpha_{3}}{3 \alpha_{4}+2 \alpha_{5}}} \frac{1}{t-\left(3 \alpha_{3} \omega^{2}-2 \alpha_{1} \omega\right) x+\xi_{0}} \\
& \times \exp [i(k x-\omega t)] .
\end{aligned}
$$

3.2. Using the First Integral Method to Solve (1). The first integral of (17) can be assumed in the following form:

$$
F(X(\xi), Y(\xi))=\sum_{i=0}^{m} a_{i}(X(\xi)) Y^{i}(\xi)=0
$$

where $a_{i}(X)(i=0,1,2, \ldots, m)$ are polynomials of $X$ and $a_{m}(X) \neq 0$. We start our analysis by assuming $m=1$ in (27).

According to the Division Theorem, there exists a polynomial $\alpha(X)+\beta(X) Y$ in $C[X, Y]$ such that

$$
\begin{aligned}
& \left(\dot{a}_{0}(X)+\dot{a}_{1}(X) Y\right) Y+a_{1}(X) \dot{Y} \\
& \quad=(\alpha(X)+\beta(X) Y)\left(a_{0}(X)+a_{1}(X) Y\right)
\end{aligned}
$$

By equating the coefficients of $Y^{j}(j=0,1,2)$ on both sides of (28), we have

$$
\begin{gathered}
\dot{a}_{1}(X)=\beta(X) a_{1}(X), \\
\dot{a}_{0}(X)=\alpha(X) a_{1}(X)+\beta(X) a_{0}(X), \\
\alpha(X) a_{0}(X)=a_{1}(X)\left(-A X-B X^{3}\right) .
\end{gathered}
$$

Through careful analysis, we find that

$$
\begin{gathered}
a_{1}(X)=1, \quad \alpha(X)=\alpha_{1} X+\alpha_{2}, \\
a_{0}(X)=\frac{\alpha_{1}}{2} X^{2}+\alpha_{2} X+\alpha_{3},
\end{gathered}
$$

where $\alpha_{3}$ is an arbitrary constant of integration. Substituting $a_{0}(X), a_{1}(X)$, and $\alpha(X)$ into (31) and setting all the coefficients of $X^{j}$ to zero, one obtains a system of nonlinear algebraic equations. Then, solving the algebraic equations, we get the following.

Case 1. One has

$$
\alpha_{1}=\sqrt{-2 B}, \quad \alpha_{2}=0, \quad \alpha_{3}=-\frac{A}{\sqrt{-2 B}} .
$$

Case 2. One has

$$
\alpha_{1}=-\sqrt{-2 B}, \quad \alpha_{2}=0, \quad \alpha_{3}=\frac{A}{\sqrt{-2 B}} .
$$

Subsequently, we obtain the following solutions:

$$
\begin{aligned}
\psi_{2,1}(x, t)= & \sqrt{-\frac{A}{B}} \operatorname{coth}\left[\sqrt{\frac{A}{2}}\left(t-\lambda x+\xi_{0}\right)\right] \\
& \times \exp [i(k x-\omega t)] \\
\psi_{2,2}(x, t)= & \sqrt{\frac{A}{B}} \tan \left[-\sqrt{-\frac{A}{2}}\left(t-\lambda x+\xi_{0}\right)\right] \\
& \times \exp [i(k x-\omega t)], \\
\psi_{2,3}= & \sqrt{-\frac{6 \alpha_{3}}{3 \alpha_{4}+2 \alpha_{5}} \overline{t-\left(3 \alpha_{3} \omega^{2}-2 \alpha_{1} \omega\right) x+\xi_{0}}} \\
& \times \exp [i(k x-\omega t)] . \\
\psi_{2,4}(x, t)= & \sqrt{-\frac{A}{B}} \tanh \left[-\sqrt{\frac{A}{2}}\left(t-\lambda x+\xi_{0}\right)\right] \\
& \times \exp [i(k x-\omega t)], \\
& \times \exp [i(k x-\omega t)] . \\
\psi_{2,6}= & \sqrt{\frac{A}{B} \cot \left[-\sqrt{-\frac{A}{2}}\left(t-\lambda x+\xi_{0}\right)\right]} \\
\psi_{2,5}(x, t)=\frac{6 \alpha_{3}}{3 \alpha_{4}+2 \alpha_{5}} \overline{t-\left(3 \alpha_{3} \omega^{2}-2 \alpha_{1} \omega\right) x+\xi_{0}} & \\
& -\omega t)],
\end{aligned}
$$

3.3. Using the Direct Integral Method to Solve (1). Multiplying (17) by $u^{\prime}$ and integrating (17) with respect to $\xi$ yield

$$
u^{\prime 2}=C-\frac{A}{2} u^{2}-\frac{B}{4} u^{4}
$$

where $C$ is an integration constant. In [24], Lai et al. have given the solutions of the following nonlinear ODE with fourdegree term:

$$
F^{\prime 2}=C_{0}+C_{2} F^{2}+C_{4} F^{4}
$$

Comparing the coefficient of (36) with that of (37), we obtain the exact travelling wave solutions for (1) as follows.

When $C=1,\left(\lambda+2 \alpha_{1} \omega-3 \alpha_{3} \omega^{2}\right) / \alpha_{3}=\left(1+m^{2}\right),\left(3 \alpha_{4}+\right.$ $\left.2 \alpha_{5}\right) / 3 \alpha_{3}=-2 m^{2}$

$$
\psi_{3,1}(x, t)=\operatorname{sn}(\xi, m) \exp [i(k x-\omega t)] .
$$

When $C=1-m^{2},\left(\lambda+2 \alpha_{1} \omega-3 \alpha_{3} \omega^{2}\right) / \alpha_{3}=(1-$ $\left.2 m^{2}\right),\left(3 \alpha_{4}+2 \alpha_{5}\right) / 3 \alpha_{3}=2 m^{2}$,

$$
\psi_{3,2}(x, t)=c n(\xi, m) \exp [i(k x-\omega t)] .
$$

When $C=m^{2}-1,\left(\lambda+2 \alpha_{1} \omega-3 \alpha_{3} \omega^{2}\right) / \alpha_{3}=\left(m^{2}-2\right),\left(3 \alpha_{4}+\right.$ $\left.2 \alpha_{5}\right) / 3 \alpha_{3}=2$,

$$
\psi_{3,3}(x, t)=d n(\xi, m) \exp [i(k x-\omega t)] .
$$


When $C=m^{2},\left(\lambda+2 \alpha_{1} \omega-3 \alpha_{3} \omega^{2}\right) / \alpha_{3}=\left(1+m^{2}\right),\left(3 \alpha_{4}+\right.$ $\left.2 \alpha_{5}\right) / 3 \alpha_{3}=-2$,

$$
\psi_{3,4}(x, t)=n s(\xi, m) \exp [i(k x-\omega t)] .
$$

When $C=-m^{2},\left(\lambda+2 \alpha_{1} \omega-3 \alpha_{3} \omega^{2}\right) / \alpha_{3}=-\left(2 m^{2}-\right.$ 1), $\left(3 \alpha_{4}+2 \alpha_{5}\right) / 3 \alpha_{3}=-2\left(m^{2}-1\right)$,

$$
\psi_{3,5}(x, t)=n c(\xi, m) \exp [i(k x-\omega t)] .
$$

When $C=-1,\left(\lambda+2 \alpha_{1} \omega-3 \alpha_{3} \omega^{2}\right) / \alpha_{3}=-\left(2-m^{2}\right),\left(3 \alpha_{4}+\right.$ $\left.2 \alpha_{5}\right) / 3 \alpha_{3}=-2\left(m^{2}-1\right)$,

$$
\psi_{3,6}(x, t)=n d(\xi, m) \exp [i(k x-\omega t)] .
$$

When $C=1,\left(\lambda+2 \alpha_{1} \omega-3 \alpha_{3} \omega^{2}\right) / \alpha_{3}=-\left(2-m^{2}\right),\left(3 \alpha_{4}+\right.$ $\left.2 \alpha_{5}\right) / 3 \alpha_{3}=-2\left(1-m^{2}\right)$,

$$
\psi_{3,7}(x, t)=s c(\xi, m) \exp [i(k x-\omega t)] .
$$

When $C=1,\left(\lambda+2 \alpha_{1} \omega-3 \alpha_{3} \omega^{2}\right) / \alpha_{3}=-\left(2 m^{2}-1\right),\left(3 \alpha_{4}+\right.$ $\left.2 \alpha_{5}\right) / 3 \alpha_{3}=-2 m^{2}\left(m^{2}-1\right)$,

$$
\psi_{3,8}(x, t)=s d(\xi, m) \exp [i(k x-\omega t)] .
$$

When $C=1-m^{2},\left(\lambda+2 \alpha_{1} \omega-3 \alpha_{3} \omega^{2}\right) / \alpha_{3}=-(2-$ $\left.m^{2}\right),\left(3 \alpha_{4}+2 \alpha_{5}\right) / 3 \alpha_{3}=-2$,

$$
\psi_{3,9}(x, t)=c s(\xi, m) \exp [i(k x-\omega t)] .
$$

When $C=-m^{2}\left(1-m^{2}\right),\left(\lambda+2 \alpha_{1} \omega-3 \alpha_{3} \omega^{2}\right) / \alpha_{3}=-\left(2 m^{2}-\right.$ 1), $\left(3 \alpha_{4}+2 \alpha_{5}\right) / 3 \alpha_{3}=-2$,

$$
\psi_{3,10}(x, t)=d s(\xi, m) \exp [i(k x-\omega t)] .
$$

When $C=-\left(m^{2}-1\right)^{2} / 4,\left(\lambda+2 \alpha_{1} \omega-3 \alpha_{3} \omega^{2}\right) / \alpha_{3}=-\left(m^{2}+\right.$ 1)/2, $\left(3 \alpha_{4}+2 \alpha_{5}\right) / 3 \alpha_{3}=1 / 2$,

$$
\psi_{3,11}(x, t)=[m c n(\xi, m) \pm d n(\xi, m)] \exp [i(k x-\omega t)] .
$$

When $C=1 / 4,\left(\lambda+2 \alpha_{1} \omega-3 \alpha_{3} \omega^{2}\right) / \alpha_{3}=-(1-$ $\left.2 m^{2}\right) / 2,\left(3 \alpha_{4}+2 \alpha_{5}\right) / 3 \alpha_{3}=-1 / 2$,

$$
\psi_{3,12}(x, t)=\frac{1 \pm c n(\xi, m)}{s n(\xi, m)} \exp [i(k x-\omega t)] .
$$

When $C=\left(1-m^{2}\right) / 4,\left(\lambda+2 \alpha_{1} \omega-3 \alpha_{3} \omega^{2}\right) / \alpha_{3}=-\left(m^{2}+\right.$ 1) $/ 2,\left(3 \alpha_{4}+2 \alpha_{5}\right) / 3 \alpha_{3}=\left(m^{2}-1\right) / 2$,

$$
\psi_{3,13}(x, t)=\frac{1 \pm s n(\xi, m)}{c n(\xi, m)} \exp [i(k x-\omega t)] .
$$

When $C=m^{2} / 4,\left(\lambda+2 \alpha_{1} \omega-3 \alpha_{3} \omega^{2}\right) / \alpha_{3}=-\left(m^{2}-\right.$ 2) $/ 2,\left(3 \alpha_{4}+2 \alpha_{5}\right) / 3 \alpha_{3}=-1 / 2$,

$$
\psi_{3,14}(x, t)=\frac{1 \pm d n(\xi, m)}{\operatorname{sn}(\xi, m)} \exp [i(k x-\omega t)] .
$$

When $C=m^{2} / 4,\left(\lambda+2 \alpha_{1} \omega-3 \alpha_{3} \omega^{2}\right) / \alpha_{3}=-\left(m^{2}-\right.$ 2) $/ 2,\left(3 \alpha_{4}+2 \alpha_{5}\right) / 3 \alpha_{3}=-m^{2} / 2$,

$$
\psi_{3,15}(x, t)=[\operatorname{sn}(\xi, m) \pm i c n(\xi, m)] \exp [i(k x-\omega t)] .
$$

When $C=1 / 4,\left(\lambda+2 \alpha_{1} \omega-3 \alpha_{3} \omega^{2}\right) / \alpha_{3}=-\left(m^{2}-\right.$ 2) $/ 2,\left(3 \alpha_{4}+2 \alpha_{5}\right) / 3 \alpha_{3}=-m^{2} / 2$,

$$
\psi_{3,16}(x, t)=\frac{s n(\xi, m)}{1 \pm d n(\xi, m)} \exp [i(k x-\omega t)]
$$

When $C=\left(m^{2}-1\right) / 4,\left(\lambda+2 \alpha_{1} \omega-3 \alpha_{3} \omega^{2}\right) / \alpha_{3}=-\left(m^{2}+\right.$ 1)/2, $\left(3 \alpha_{4}+2 \alpha_{5}\right) / 3 \alpha_{3}=\left(1-m^{2}\right) / 2$,

$$
\psi_{3,17}(x, t)=\frac{d n(\xi, m)}{1 \pm m s n(\xi, m)} \exp [i(k x-\omega t)] .
$$

When $C=\left(1-m^{2}\right) / 4,\left(\lambda+2 \alpha_{1} \omega-3 \alpha_{3} \omega^{2}\right) / \alpha_{3}=-\left(m^{2}+\right.$ 1)/2, $\left(3 \alpha_{4}+2 \alpha_{5}\right) / 3 \alpha_{3}=\left(m^{2}-1\right) / 2$,

$$
\psi_{3,18}(x, t)=\frac{c n(\xi, m)}{1 \pm s n(\xi, m)} \exp [i(k x-\omega t)]
$$

Remark 2. When the modulus $m \rightarrow 1$, Jacobi elliptic function $\operatorname{sn}(\xi, m) \rightarrow \tanh (\xi), c n(\xi, m) \rightarrow \operatorname{sech}(\xi)$, and $d n(\xi) \rightarrow$ $\operatorname{sech}(\xi)$. Hence, we can obtain the hyperbolic function solutions. For example, $\psi_{3,1}$ and $\psi_{3,2}$ are turned into the kink type envelope wave solution

$$
\begin{gathered}
\psi_{3,1,1}=\exp [i(k x-\omega t)] \tanh \left(t-\lambda x+\xi_{0}\right), \\
A=2, \quad B=-2
\end{gathered}
$$

and the bell type envelope wave solution

$$
\begin{gathered}
\psi_{3,2,1}=\exp [i(k x-\omega t)] \operatorname{sech}\left(t-\lambda x+\xi_{0}\right), \\
A=-1, \quad B=2 .
\end{gathered}
$$

Formula (57) is the same as the solution $\psi_{1,2}$.

In particular, $\psi_{3,4}, \psi_{3,12}$, and $\psi_{3,15}$ degenerate into

$$
\begin{aligned}
\psi_{3,4,1}= & \exp [i(k x-\omega t)] \operatorname{coth}\left(t-\lambda x+\xi_{0}\right), \\
A=2, \quad B=-2, & \\
\psi_{3,12,1}= & {\left[\operatorname{coth}\left(t-\lambda x+\xi_{0}\right)+\operatorname{csch}\left(t-\lambda x+\xi_{0}\right)\right] } \\
& \times \exp [i(k x-\omega t)], \quad A=\frac{1}{2}, \quad B=-\frac{1}{2}, \\
\psi_{3,15,1}= & {\left[\tanh \left(t-\lambda x+\xi_{0}\right)+i \operatorname{sech}\left(t-\lambda x+\xi_{0}\right)\right] } \\
& \times \exp [i(k x-\omega t)], \quad A=\frac{1}{2}, \quad B=-\frac{1}{2},
\end{aligned}
$$

respectively, which are in full agreement with the solutions $\psi_{1,1}, \psi_{1,4}$, and $\psi_{1,3}$ obtained above.

In addition, $\psi_{3,3}$ and $\psi_{3,9}$ degenerate into the known solutions (32) and (33) in [23], respectively. 
Remark 3. When $m \rightarrow 0, \operatorname{sn}(\xi, m) \rightarrow \sin (\xi), c n(\xi, m) \rightarrow$ $\cos (\xi)$, and $d n(\xi) \rightarrow 1$. In this case, we mention $\psi_{3,7}, \psi_{3,9}$, $\psi_{3,12}$, and $\psi_{3,13}$ which degenerate into

$$
\begin{aligned}
& \psi_{3,7,0}=\exp [i(k x-\omega t)] \tan \left(t-\lambda x+\xi_{0}\right), \\
& A=-2, \quad B=-2, \\
& \psi_{3,9,0}=\exp [i(k x-\omega t)] \cot \left(t-\lambda x+\xi_{0}\right), \\
& A=-2, \quad B=-2, \\
& \psi_{3,12,0}=\left[\csc \left(t-\lambda x+\xi_{0}\right)+\cot \left(t-\lambda x+\xi_{0}\right)\right] \\
& \times \exp [i(k x-\omega t)], \quad A=-\frac{1}{2}, \quad B=-\frac{1}{2}, \\
& \psi_{3,13,0}=\left[\sec \left(t-\lambda x+\xi_{0}\right)-\tan \left(t-\lambda x+\xi_{0}\right)\right] \\
& \times \exp [i(k x-\omega t)], \quad A=-\frac{1}{2}, \quad B=-\frac{1}{2},
\end{aligned}
$$

respectively, which are in agreement with the solutions $\psi_{1,6}$, $\psi_{1,7}, \psi_{1,9}$, and $\psi_{1,8}$. It is worth mentioning that $\psi_{3,4}$ and $\psi_{3,5}$ degenerate into the known solutions obtained by Liu (formulae (37) and (36) in [23]).

Remark 4. With the aid of Mathematica, we have checked the solutions $\psi_{i, j}(i=1,2,3, j=1,2, \ldots)$ by putting them back into (1).

Remark 5. Compared with the work in $[4,23]$, we obtained more soliton solutions, triangle periodic solutions, and Jacobi elliptic solutions including some known solutions in $[4,23]$.

\section{Discussion and Conclusion}

In summary, we investigate the travelling wave solutions to nonlinear Schrödinger equation with a higher-order dispersive term by using three methods, namely, the auxiliary equation method, the first integral method, and the direct integral method. As a result, exact solutions including Jacobi elliptic function solution, the kink type envelope wave solution, and the bell type envelope wave solution are obtained. In particular, Jacobi elliptic function solutions degenerate into the hyperbolic function solutions and the triangular function solutions obtained via the auxiliary equation method and the first integral method. Because the nonlinear Schrödinger equation with a higher-order dispersive term is an important model in physics science, these results are expected to help understand wave dynamics in the study of ultrashort femtosecond pulses in optical fibres.

\section{Acknowledgments}

The authors would like to express their thanks to the referees for their useful comments which led to some improvements of the current paper. This work is supported in part by the National Natural Science Foundation of China (no. 11071141) and the Natural Science Foundation of Shandong Province of China (ZR2011AL018 and ZR2011AQ008).

\section{References}

[1] W.-X. Ma and M. Chen, "Direct search for exact solutions to the nonlinear Schrödinger equation," Applied Mathematics and Computation, vol. 215, no. 8, pp. 2835-2842, 2009.

[2] M. J. Ablowitz and H. Segur, Solitons and the Inverse Scattering Transform, vol. 4, Society for Industrial and Applied Mathematics (SIAM), Philadelphia, Pa, USA, 1981.

[3] A. Hasegawa and Y. Kodama, Solitons in Optical Communications, Oxford University Press, New York, NY, USA, 1995.

[4] L. X. Li and M. L. Wang, "The $\left(G^{\prime} / G\right)$-expansion method and travelling wave solutions for a higher-order nonlinear Schrödinger equation," Applied Mathematics and Computation, vol. 208, no. 2, pp. 440-445, 2009.

[5] W.-X. Ma and J.-H. Lee, "A transformed rational function method and exact solutions to the $3+1$ dimensional JimboMiwa equation," Chaos, Solitons \& Fractals, vol. 42, no. 3, pp. 1356-1363, 2009.

[6] F. X. Wu and Z. D. Dai, "New bright and dark solitons for quintic nonlinear derivative Schrödinger equation," Applied Mathematics and Computation, vol. 218, no. 18, pp. 9305-9309, 2012.

[7] W. X. Ma, "Bilinear equations, Bell polynomials and linear superposition princ," Journal of Physics, vol. 411, Article ID iple012021, 2013.

[8] Y. Liu, "Exact solutions to nonlinear Schrödinger equation with variable coefficients," Applied Mathematics and Computation, vol. 217, no. 12, pp. 5866-5869, 2011.

[9] N. Taghizadeh and M. Mirzazadeh, "The simplest equation method to study perturbed nonlinear Schrödinger's equation with Kerr law nonlinearity," Communications in Nonlinear Science and Numerical Simulation, vol. 17, no. 4, pp. 1493-1499, 2012.

[10] N. A. Kudryashov, "On types of nonlinear nonintegrable equations with exact solutions," Physics Letters A, vol. 155, no. 4-5, pp. 269-275, 1991.

[11] R. Hirota, The Direct Method in Soliton Theory, vol. 155, Cambridge University Press, Cambridge, UK, 2004.

[12] A. M. Wazwaz, "On multiple soliton solutions for coupled kdvmkdv equation," Nonlinear Science Letters A, vol. 1, no. 3, pp. 289-296, 2010.

[13] R. Hirota, "Direct method of finding exact solutions of nonlinear evolution equations," in Bäcklund transformations, R. Bullough and P. Caudrey, Eds., Springer, Berlin, Germany, 1980.

[14] A.-M. Wazwaz, "A sine-cosine method for handling nonlinear wave equations," Mathematical and Computer Modelling, vol. 40, no. 5-6, pp. 499-508, 2004.

[15] A.-M. Wazwaz, "The tanh method for traveling wave solutions of nonlinear equations," Applied Mathematics and Computation, vol. 154, no. 3, pp. 713-723, 2004.

[16] E. G. Fan, "Extended tanh-function method and its applications to nonlinear equations," Physics Letters A, vol. 277, no. 4-5, pp. 212-218, 2000.

[17] J.-H. He and X.-H. Wu, "Exp-function method for nonlinear wave equations," Chaos, Solitons \& Fractals, vol. 30, no. 3, pp. 700-708, 2006.

[18] W.-X. Ma and Z. Zhu, "Solving the $(3+1)$-dimensional generalized KP and BKP equations by the multiple exp-function algorithm," Applied Mathematics and Computation, vol. 218, no. 24, pp. 11871-11879, 2012. 
[19] E. G. Fan and J. Zhang, "Applications of the Jacobi elliptic function method to special-type nonlinear equations," Physics Letters A, vol. 305, no. 6, pp. 383-392, 2002.

[20] Z. S. Feng, "The first-integral method to study the BurgersKorteweg-de Vries equation," Journal of Physics A, vol. 35, no. 2, pp. 343-349, 2002.

[21] W.-X. Ma, "Comment on the $3+1$ dimensional KadomtsevPetviashvili equations," Communications in Nonlinear Science and Numerical Simulation, vol. 16, no. 7, pp. 2663-2666, 2011.

[22] F. D. Xie, Y. Zhang, and Z. S. Lü, "Symbolic computation in nonlinear evolution equation: application to $(3+1)$-dimensional Kadomtsev-Petviashvili equation," Chaos, Solitons and Fractals, vol. 24, no. 1, pp. 257-263, 2005.

[23] C. P. Liu, "Exact solutions for the higher-order nonlinear Schördinger equation in nonlinear optical fibres," Chaos, Solitons and Fractals, vol. 23, no. 3, pp. 949-955, 2005.

[24] S. Y. Lai, X. M. Lv, and M. Y. Shuai, "The Jacobi elliptic function solutions to a generalized Benjamin-Bona-Mahony equation," Mathematical and Computer Modelling, vol. 49, no. 1-2, pp. 369378, 2009. 


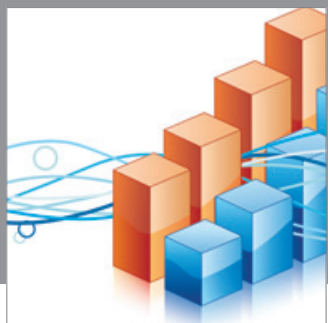

Advances in

Operations Research

mansans

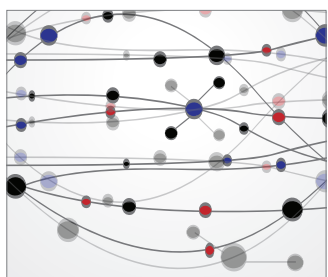

The Scientific World Journal
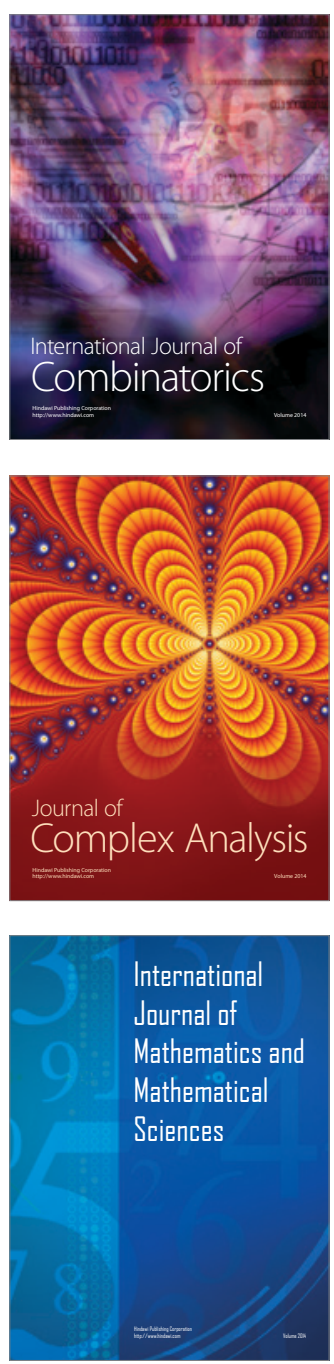
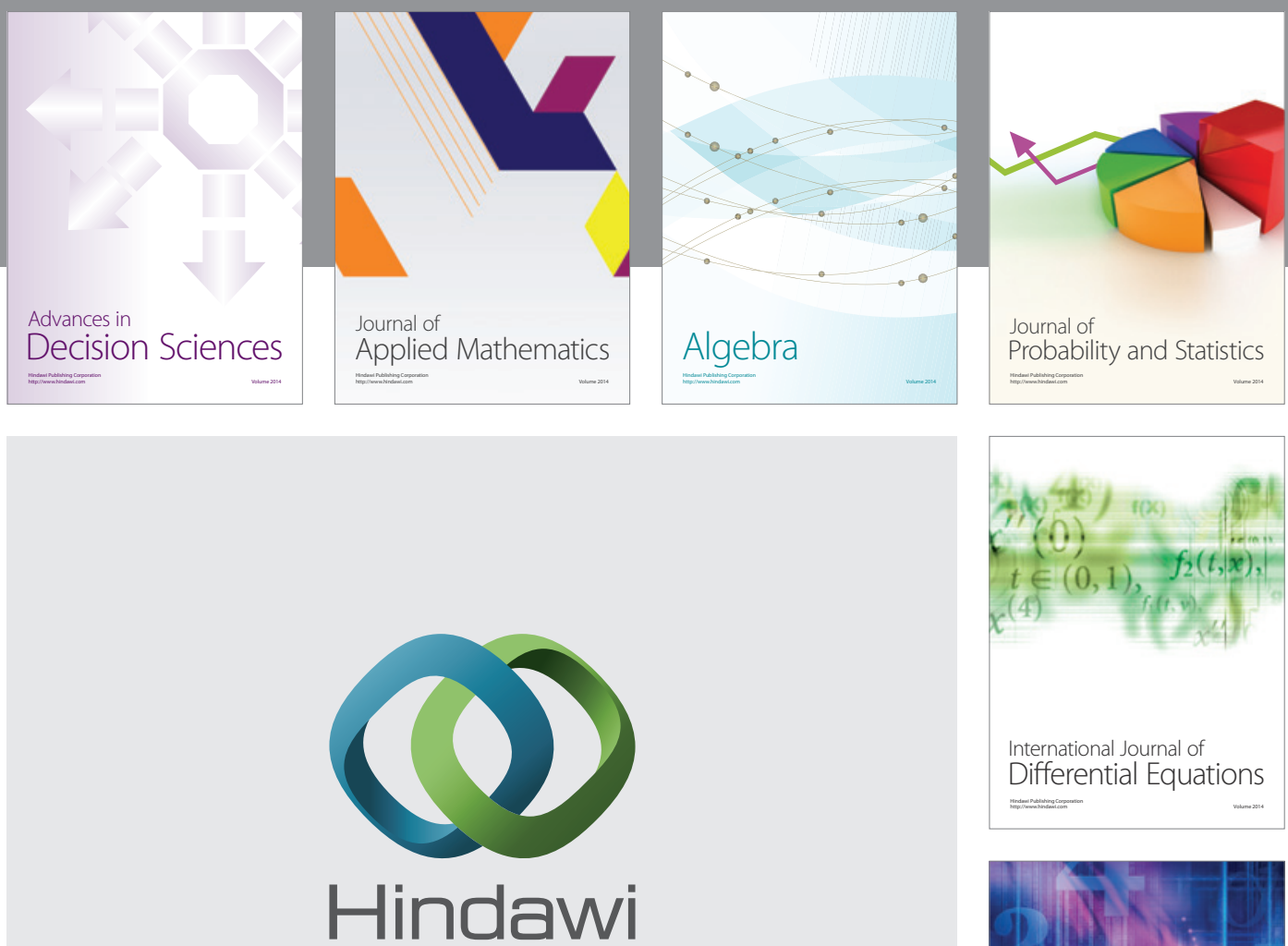

Submit your manuscripts at http://www.hindawi.com
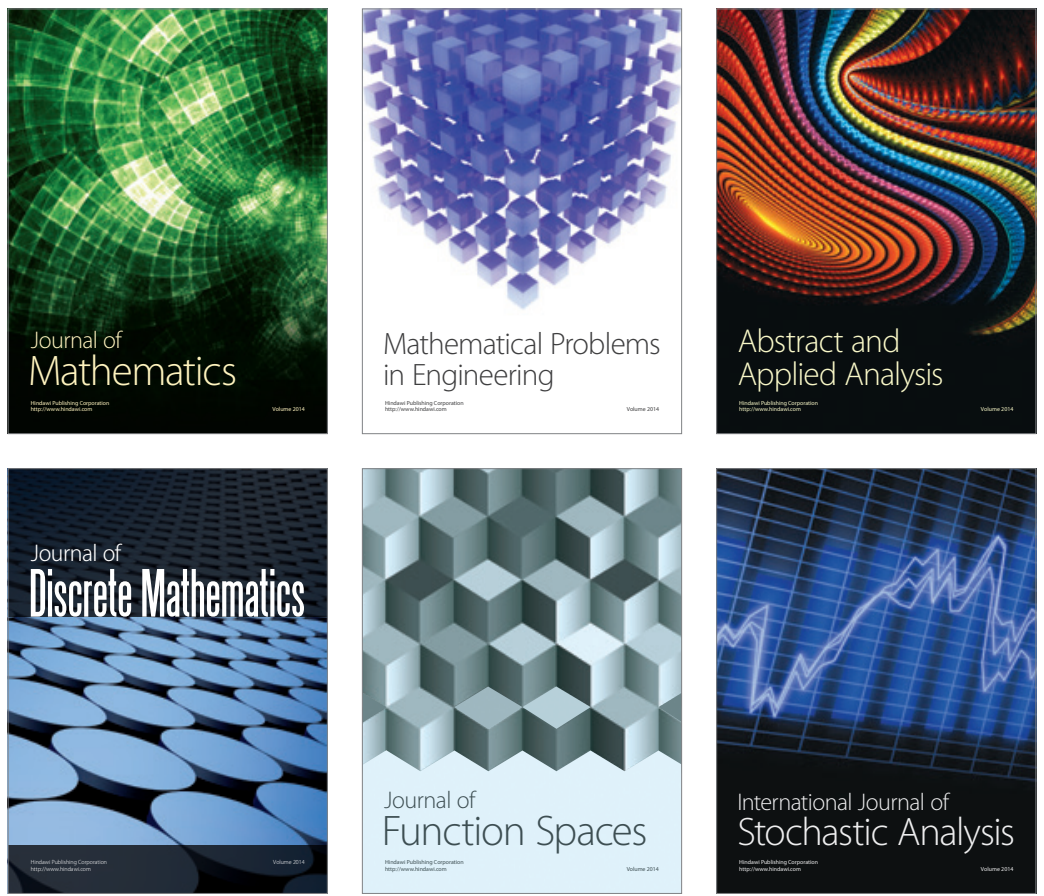

Journal of

Function Spaces

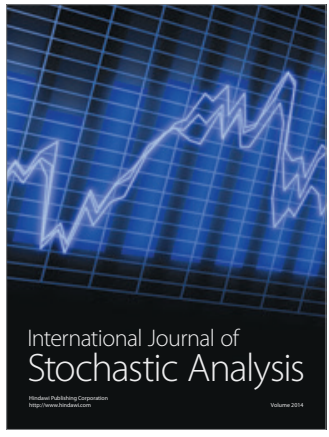

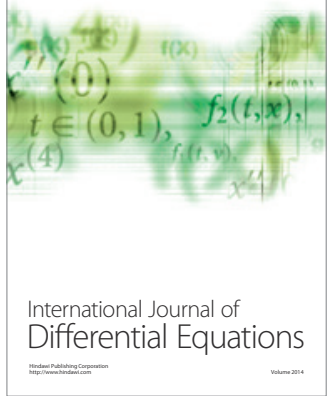
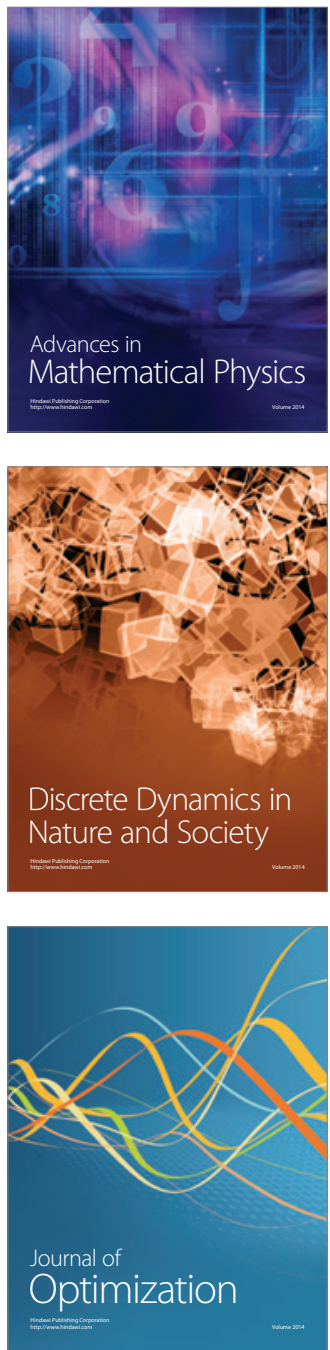